\begin{tabular}{|c|c|}
\hline Title & A scidian sperm receptor attached to the vitelline coat during oocyte maturation \\
\hline Author(s) & Sawada, H.; Tanaka, E.; A be, Y.; Takizawa, S.; Takahashi, Y.; Fujino, J.; Y okosawa, H. \\
\hline Citation & ZYGOTE, 8, S14S15 \\
\hline Issue Date & 2000 \\
\hline Doc URL & http:/hdl .handle.net/2115/6116 \\
\hline Type & article \\
\hline Note & $\begin{array}{l}\text { Supplement : } \\
\text { Copyright } \odot \text { (2000) Cambridge University Press }\end{array}$ \\
\hline File Information & ZYGOTE8.pdf \\
\hline
\end{tabular}

Instructions for use 


\title{
Ascidian sperm receptor attached to the vitelline coat during oocyte maturation
}

\author{
Hitoshi Sawada ${ }^{1}$ Etsuko Tanaka ${ }^{1}$, Yukichi Abe ${ }^{1}$, Satoshi Takizawa ${ }^{2}$, Youko Takahashi ${ }^{1}$, \\ Junko Fujino ${ }^{1}$ and Hideyoshi Yokosawa ${ }^{1}$ \\ ${ }^{1}$ Department of Biochemistry, Graduate School of Pharmaceutical Sciences, Hokkaido University, Sapporo 060-0812, Japan \\ ${ }^{2}$ Department of Life Science, Tokyo Institute of Technology, Nagatsuta Yokohama 226-8501, Japan
}

While all ascidians (Urochordata) are hermaphroditic, some, including Halocynthia roretzi (Fuke, 1983) and Ciona intestinalis (Rosati \& De Santis, 1978) are strictly self-sterile because of a self-nonself recognition system in the interaction between the sperm and the vitelline coat of the eggs. However, immature oocytes (Fuke \& Numakunai, 1996) and acidic-seawater-treated mature oocytes (Morgan, 1939; Kawamura et al., 1991) are selffertile. It is thought that a putative self-nonself recognition molecule, which is detached or modified by treatment with acidic seawater, may be attached to the vitelline coat during oocyte maturation. Although the existence of a self-nonself recognition system in the fertilisation process is well known, the molecular entity has yet to be conclusively identified. However, there have been several attempts to identify such a molecule in Ciona (Marino et al., 1999). In the present study, we have isolated and analysed a molecule which appears to be responsible for allorecognition in the interaction between sperm and eggs of the ascidian Halocynthia roretzi.

\section{Materials and methods}

Biologicals. A solitary ascidian Halocynthia roretzi Type $C$ was used in this study. The fertilisation experiment was carried out as described previously (Sawada et al., 1982).

Isolation and N-terminal Sequencing of HrVC70. Vitelline coats were isolated from immature and mature oocytes of the ascidian by homogenisation and repeated washing with $5 x$ diluted artificial seawater. The isolated vitelline coats were subjected to SDS-PAGE, followed by blotting to a PVDF membrane. The $N$-terminal amino acid sequence of the $70 \mathrm{kDa}$ main component (HrVC70) was determined by a protein sequencer.

Cloning of HrVC70-precursor cDNA. Based on the $N$-terminal sequence, degenerate primers were designed and a polymerase chain reaction (PCR) was carried out. The PCR product was used as a probe for screening the $H$. roretzi gonad cDNA library to isolate the HrVC70 cDNA clone.

Sperm binding assay to HrVC70-agarose. The isolated HrVC70 was coupled to Affi-Gel-10 beads. The HrVC70-agarose beads thus obtained were incubated with sperm suspended in artificial seawater, followed by washing. The number of sperm bound to the HrVC70-agarose bead was counted after incubation with DAPI under a fluorescence microscope using UV excitation.

Molecular mass estimation. The molecular mass of HrVC70 was estimated by a MALDI-TOF mass spectrometry.

Nucleotide sequence analysis of HrVC70 in each individual. RNA was prepared from the gonad of each individual, and a reverse transcription (RT)-PCR was carried out using the same protocols.

\section{Results}

Attachment of the HrVC70 molecule to the vitelline coat during ascidian oocyte maturation and its detachment by treatment with acid. Since mature oocytes, unlike immature oocytes, are self-sterile (Fuke \& Numakunai, 1996), it is thought that they might acquire a self-nonself recognition system during oocyte maturation. If this is the case, a putative allorecognition molecule may be attached to the vitelline coat during oocyte maturation.

To assess this possibility, we isolated the vitelline coats from immature and mature oocytes and compared their SDS-PAGE patterns. The results showed that the amount of a $70 \mathrm{kDa}$ main component ( $\mathrm{HrVC70})$ in the vitelline coat obtained from mature oocytes was much higher than that of immature ooyctes. This suggests that $\mathrm{HrVC70}$ becomes associated with the vitelline coat during oocyte maturation. 
Next, we investigated whether HrVC70 becomes detached from the vitelline coat by acid extraction. SDS-PAGE analysis revealed that the $\mathrm{HrVC70}$ is released from isolated vitelline coats by treatment with $1 \mathrm{mM} \mathrm{HCl}$ (pH 3) but not with slightly alkaline Tris buffer $(\mathrm{pH} 8)$. From these results, we suggest that $\mathrm{HrVC70}$ is a candidate molecule involved in self-nonself recognition at fertilisation.

Sperm receptor activity of $\mathrm{HrVC70}$. To investigate whether $\mathrm{HrVC70}$ has a sperm-receptor activity, the number of sperm bound to a single $\mathrm{HrVC70}$-agarose bead was counted. Under the conditions tested, sperm were able to bind to the $\mathrm{HrVC70}$ beads. In addition, the binding ability of nonself sperm to a $\mathrm{HrVC70-agarose}$ bead appeared to be higher than that of the self sperm, suggesting that $\mathrm{HrVC70}$ is a candidate sperm receptor responsible for self-nonself recognition.

Isolation of the cDNA of $\mathrm{HrVC70}$ and its structural analysis. A cDNA clone of $\mathrm{HrVC70}$ precursor ( $\mathrm{HrVC120}$ ) was isolated and its amino acid sequence deduced. The isolated clone revealed that HrVC120 has an open reading frame of 1163 amino acids with an estimated molecular mass of $124498 \mathrm{Da}$. This HrVC120 consists of the Nterminal signal sequence, 13 EGF-like repeats and a ZP homologous domain, which is followed by a single transmembrane domain at the $\mathrm{C}$-terminal region. Fhis precursor protein appears to be processed by a trypsinlike protease at the Arg-668 residue located between the twelfth and thirteenth EGF-like repeats, which is estimated from the results of C-terminal sequence analysis and of mass spectrometry (68 $800 \mathrm{Da})$.

Variability in the amino acid sequence of HrVC70 in each individual. If $\mathrm{HrVC70}$ is involved in self-nonself recognition in gamete interactions, the amino acid sequence of HrVC70 from each individual should be different. To test this hypothesis, RNA was prepared from the gonads of 10 individuals, and RT-PCR carried out using primers corresponding to the signal sequence and the region downstream of the 12 EGF-like repeats. The PCR products were subjected to DNA sequence analysis. From these experiments it was determined that none of the HrVC70 molecules obtained from the different individuals were identical to each other, although the basic structures of HrVC70 were very similar. The point mutations appear to be restricted to a region located between each EGF-like domain, and also between the third and fourth Cys residues in each EGF-like repeat. Since $\mathrm{HrVC70}$ obtained from each individual showed a variability, it is very likely that this HrVC70 molecule is directly involved in self-nonself recognition in the interaction between sperm and the vitelline coat of the eggs.

\section{Discussion}

The present study has demonstrated the DNA sequence and the deduced amino acid sequence of the HrVC70 precursor ( $\mathrm{HrVC120}$ ) from H. roretzi. Furthermore, we found several lines of evidence indicating that $\mathrm{HrVC70}$ is involved in self-nonself recognition in the ascidian fertilisation process.

However, we did not identify the cellular source of the HrVC120 molecule. In connection with this, it is intriguing to note that the establishment of the self-nonself recognition system in oocytes of $H$. roretzi during oocyte maturation is blocked by trypsin inhibitors (Fuke \& Numakunai, 1999), and this factor involved in allorecognition is thought to be derived from follicle cells. Similarly, it is also known that the follicle cells play a key role in the acquisition of self-nonself recognition in C. intestinalis (Marino et al., 1999). Taking into account these results, it is plausible that $\mathrm{HrVC} 120$ protein may be biosynthesised and the N-terminal half containing the 12 EGF-like repeats may be processed and attached to the vitelline coat, conferring self-sterility to the eggs.

We recently found that HrVC70 is degraded by the sperm proteasome in a ubiquitin-dependent manner during fertilisation. Therefore this degradation signal may be triggered only when a particular VC70 molecule is recognised by a sperm-membrane molecule as nonself.

Further studies on the mechanism producing variability in the VC70 structures as well as on the genomic structure of VC70 are in progress in our laboratory.

\section{References}

Fuke, T.M. (1983). Rouxs Arch. Dev. Biol. 192, 347-52.

Fuke, T.M. \& Numakunai, T. (1996). Rouxs Arch. Dev. Biol. 205, 391-400.

Fuke, T.M. \& Numakunai, T. (1999). Mol. Reprod. Dev. 52, 99-106.

Kawamura, K., Nomura, T., Kameda, H., Shimamoto, H. \& Nakauchi, M. (1991). Dev. Growth Differ. 33, 139-48.

Marino, R., De Santis, R., Giuliano, P. \& Pinto, M. R. (1999). Proc. Natl. Acad. Sci. USA 96, 9633-6.

Morgan, T.H. (1939). J. Exp. Zool. 95, 37-59.

Rosati, F. \& De Santis, R.(1978). Exp. Cell Res. 112, 111-19.

Sawada, H., Yokosawa, H., Hoshi, M. \& Ishii, S. (1982). Gamete Res. 5, 291-301. 